INTRODUCTION

\title{
SECTION SPÉCIALE INTRODUCTION VIEILLISSEMENT ET SANTÉ MENTALE
}

\author{
BERNADETTE DALLAIRE \\ Université Laval
}

et

JOSEPH TINDALE

Université de Guelph

\begin{abstract}
La très grande majorité des Canadiennes et Canadiens âgés vivent sans problèmes de santé mentale majeurs. Pour une large part, ceci montre bien la stabilité du bien-être mental à travers les cycles de la vie (Chappell, Gee, McDonald, \& Stones, 2003). Cependant, certaines circonstances créent des conditions qui suscitent des besoins particuliers en matière de santé mentale. Ces besoins sont-il correctement reconnus-ou sont-ils reconnus, point? Et surtout, leur répond-on de manière adéquate? Dans un texte fondateur sur la psychiatrie et les aînés et aînées, Butler (1975) a évoqué un "nihilisme thérapeutique »—une attitude pessimiste qui serait répandue dans les services professionnels de santé mentale fournis aux aînés et aînées. Plus récemment, le Groupe de travail sur la déstigmatisation du Bureau européen de l'Organisation mondiale de la Santé a dénoncé le stigmate, la discrimination et l'exclusion qui frappent encore aujourd'hui les personnes âgées souffrant de troubles mentaux (Mendonça Lima, Levav, Jacobsson, \& Rutz, 2003). À ce titre, certains analystes soulignent que ces phénomènes trouvent leur source dans le processus de doubleétiquetage par lequel les citoyennes et citoyens âgés sont stigmatisés à la fois en raison du vieil âge et de la maladie mentale (Bartels, 2003; Dallaire, McCubbin, Carpentier, \& Clément, sous presse).
\end{abstract}

Les articles de cette section spéciale abordent les variations de l'expérience du vieillissement normal chez des personnes qui sont confrontées à des problématiques de santé mentale, des conditions sociales radicalement transformées ou des maladies chroniques. Dans chaque cas, l'attention est portée sur les interactions entre les patient(e)s/client(e)s âgés, les professionnels et professionnelles et, quelquefois, les proches-aidant(e)s. Ces textes visent à identifier et documenter certains aspects des réponses sociales et professionnelles aux besoins spécifiques des aînés et aînées en matière de santé mentale.

Le texte de Garcia et Finlayson examine les besoins de services des personnes souffrant de sclérose en plaques, à mesure qu'elles avancent en âge. Taylor, TaylorHenley et Doan discutent quant à eux sur les défis rencontrés par les immigrantes et immigrants âgés et sur les voies par lesquelles les classes d'anglais langue seconde (ALS) peuvent dépasser leur fonction première et être conçus comme leviersfacilitants dans la réponse à ces défis particuliers. La discussion sur ces classes d'ALS en tant que modèle de "meilleures pratiques" met aussi en lumière leur potentiel en matière de promotion de la santé, à travers le développement d'outils permettant de faire face à différentes problématiques rencontrées par les immigrantes et immigrants âgés. L’Outil d'évaluation des politiques en matière de santé mentale des aînés dont il 
est question dans l'article de MacCourt et Tuokko est un instrument d'analyse destiné aux personnes âgées, aux professionnels et professionnelles et aux décideurs impliqués dans les processus de construction des programmes et politiques publiques. L'objectif de cet outil est d'insérer la prévention des problèmes de santé mentale et la promotion du bien-être mental dans la conception et la mise en œuvre des programmes et des politiques. À un niveau différent, Pérodeau, Paradis, Ducharme, Cohen et Collin introduisent la dimension familiale dans la prestation des services: ils montrent l'importance du soutien des proches dans le bien-être ou la détresse psychologique perçus chez les femmes âgées utilisatrices de médicaments psychotropes; en particulier, ces auteurs mettent en évidence l'impact des attitudes des proches-aidantes à l'égard de l'utilisation de telles médications (ou de la cessation de cette utilisation) et donc leur influence sur le cours et les résultats des interventions médicales de santé mentale.

Toutes ces recherches contribuent à faire progresser notre compréhension des conditions de vie, des services professionnels et du soutien apporté par les proches/ non-professionnel(le)s. Toutefois, l'examen de ces questions doit, d'évidence, se poursuivre. Comme les articles réunis ici le montrent éloquemment, cet examen ne doit pas passer exclusivement par une approche clinique, mais doit aussi faire appel à des lectures psychosociales et macrosociales afin de rendre compte, de solutionner et ultimement de contrecarrer le «nihilisme » dont on soupçonne la présence en ce qui concerne les problématiques de santé mentale touchant les aînés et aînées.

\section{RÉFÉRENCES}

Bartels, S.J. (2003). Improving the United States' system of care for older adults with mental illness: Findings and recommendations for the President's New Freedom Commission on Mental Health. American Journal of Geriatric Psychiatry, 11, 486-497.

Butler, R.N. (1975). Psychiatry and the elderly: An overview. American Journal of Psychiatry, 132, 893-900.

Chappell, N., Gee, E., McDonald, L., \& Stones, M. (2003). Aging in contemporary Canada. Toronto: Prentice Hall.

Dallaire, B., McCubbin, M., Carpentier, N., \& Clément, M. (sous presse). Representations of elderly with mental health problems held by psychosocial practitioners from community and institutional settings. Dans S. Dumont \& M. St-Onge (dir.), Social work in health and mental health. New York: Haworth Press.

Mendonça Lima, C.A., Levav, I., Jacobsson, L., \& Rutz, W. (2003). Stigma and discrimination against older people with mental disorders in Europe. International Journal of Geriatric Psychiatry, 18, 679-682. 